\title{
TOMOGRAPHIC RECONSTRUCTION OF MICRO-VASCULAR NETWORK IN CEREBRAL CORTICAL SAMPLES
}

Kristóf Kapitány ${ }^{1}$, László Négyessy ${ }^{2,3}$, Árpád Barsi ${ }^{1}$

${ }^{1}$ Department of Photogrammetry and Geoinformatics, Budapest University of Technology and

Economics

${ }^{2}$ Wigner Research Centre for Physics, Hungarian Academy of Science

${ }^{3}$ Department of Anatomy, Histology and Embryology, Semmelweis University

kapitany@eik.bme.bu

\section{Abstract}

Objectives

The purpose of the research is the automatic processing and analysis of high-amount (nearly 1500) and high-resolution synchrotron based tomography images of mammalian cerebral cortical tissues. The objective was to acquire quantitative data allowing future blood flow modeling based on the structure of cerebral vessel network. The brain samples were processed with histological method compatible with synchrotron x-ray microtomography. The method developed performs automated extraction of the relevant parameters of the vessel network on a standard personal computer using previously set parameters.

Method

The method implemented on the image series performs automatic segmentation by using determined intensity values. The procedure finds the boundary of the vessels and provides quantitative measures of the segments, and it also creates the topology of the vessel network.

Results

Our method produces topologically correct network of continuous, branching micro blood vessels of the sample studied. We present multiple options for the visualization of the detected vessels. The data structure also contains the statistics of each detected cross-section, in addition with the numerical quality statistics of the continuous vessel branches (e. g. vessel length or mean diameter). The algorithm resulted in highly accurate fitting at micrometer geometric resolution.

\section{Conclusion}

The reconstructed vessel network is the geometrically accurate description of the blood vessels in the tissue. The data acquired could be the base of a blood vessel surface reconstruction, and by adding physiological information it could be used for simulations in normal and pathological conditions (e. g. arteriosclerosis). The precise vascular geometry and the distance distribution provides information about the barely understood neurovascular coupling, which is the basis of such modern diagnostic and research tools as the fMRI. After analyzing samples from multiple species or brain regions the difference could be determined objectively.

\section{Introduction}

To understand brain functioning, we have to know precisely the inner structure of the cerebral tissue, the connection network of the blood vessels and the neurons. ${ }^{1,2}$ The conventional method of medical imagery is to analyze the tissues (after special pretreatment) with the use of optical- or 
electron microscope depending on the object of examination. Traditional microscopic methods cannot detect overlapping structures which are small relative to the thickness of the tissue. Therefore the tissue must be cut for ultra-thin slices, and each thin section be analyzed separately. If vessels are the object of research the slices should be 1-2 $\mu \mathrm{m}$ thick, but in case of dendrites 50$70 \mathrm{~nm}$ thin sections must be cut, ${ }^{3}$ but sometimes even $15-40 \mathrm{~nm}$ thin cuts are needed. ${ }^{4}$ This process is extremely labor-intensive and inefficient, and as the sample is sliced, it can't be used for other analyses. X-ray micro tomography enables non-destructive tests on the tissue samples. Computer tomography (CT) creates series of projections with the radioscopy of the rotated sample; hence CT enables thorough introspection inside the examined material. By analyzing tissues using this method, after special pretreatment, various objects or materials could be detected in a sample. ${ }^{5,6}$ This article presents the process of an image series, showing and discussing the results.

The purpose of this study is to detect the micro blood vessels of the cerebral cortex of mammalian species on series of microtomographic images, and to acquire information from the image data in order to create a geometrically accurate vessel network.

\section{Materials}

X-ray micro-tomography images were obtained from samples of the mammalian cerebral cortex. The present study is focused on the examination of the rat cortical tissue. Animals were treated in accordance with the Guide for the Care and Use of Laboratory Animals (National Research Council 1996), European Directive 86/609 and the guidelines of the local institutional animal care and use committee. Histological pre-treatment was started with transcardial perfusion of an aldehyde solution to preserve brain structure. Blocks including the region of interest were removed from the fixed brain and sections were cut at 20-60 $\mu \mathrm{m}$ thickness, then washed and stained. In our case the sections were stained with phosphotungstic acid (PTA), which enhances the contrast of the tissue by binding to proteins and nucleotides. Consequently, the lumen of the blood vessels remained unstained in the background of the labeled tissue. The following step was the dehydration, before embedding into resin. Embedded tissues can be further cut into very thin, so called ultra-thin sections. Using a micropunch tool we cut a cylinder-shaped sample from the embedded section with dimensions of $500 \mu \mathrm{m}$ in diameter and $60 \mu \mathrm{m}$ in height. ${ }^{7}$

The samples were imaged at the Swiss Light Source (SLS) at the Paul Scherrer Institute in Switzerland, in a third-generation synchrotron light source, which provides photon beams of high brightness. The X-ray tomography images were created with a beamline, called TOMCAT (TOmographic Microscopy and Coherent rAdiology experimenTs), which is a special experimental instrument of the SLS. The samples were analyzed by a monochromatic beam with $10 \mathrm{keV}$ energy using the TOMCAT application, which is designed to rotate the samples therefore to provide three-dimensional data ${ }^{8}$ By the rotation of the sample holder $1401 \mathrm{X}$-ray tomography images were captured about one sample with the dimension of $2048 \times 1356$ pixels. The nominal pixel size of the images is $0.38 \mu \mathrm{m}$, which provides high-resolution images of the sample. They were transformed into $2048 \times 2048$ pixel wide cross-section images using inverse Radontransformation (Figure 1). Because the sample is transparent to the $\mathrm{X}$-ray radiation (although anisotropically as the result of PTA staining) raw images include information of the whole sample 
from different rotation angles. Radon-transformation, which needs powerful computational resources, is used to create series of digital cross sections of the sample, similar to that seen in regular CT examinations. Our methods are designed for simple personal computers and so this transformation optimizes the input data, without the loss of valuable information.

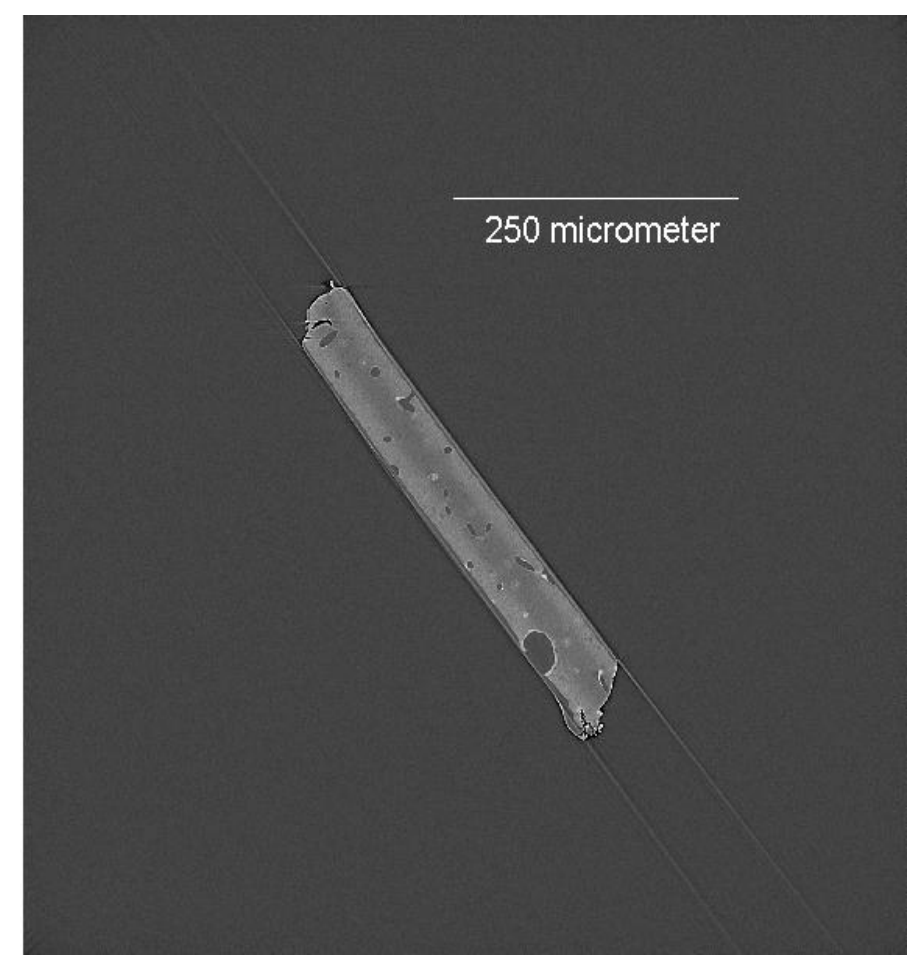

Figure 1. A single image after inverse Radon transformation

\section{Method}

\section{Detection of the vessels}

The images are of high-resolution, the color depth is 16-bit, so because of the huge data amount it is hard to work with the whole series of a block on a standard personal computer. Our method analyzes each image in the series one-by-one and creates the segments of the vessels. To reduce processing time the sample was segmented from the image, with an algorithm that finds the bounding box of the sample's cross section on each image, and trims out the union of the bounding boxes from the images. This reduced the image size from $2048 \times 2048$ to $859 \times 1096$ pixels resulting considerable less process time and memory consumption. The algorithm was written in MATLAB.

To reconstruct a blood vessel model, we have to find the vessels first. The vessels' cross-sections were segmented using a threshold value, and were saved in a stacked image format, where a layer was created for each projection as a binary image. The threshold values were determined using the Java-based, open source image processing software ImageJ . ' Using this software the image series could be loaded into one group and the threshold of the blood vessels could be determined with the stack histogram of the image group. The segmented image of Figure 1 is shown in Figure 2. During the segmentation false regions were also detected e. g. interior noisy blobs or tissue shreds on the outer surface of the sample. The surface of the sample is rough because the sampling method can't provide perfect cylinder-shaped blocks due to the soft and ductile 
biological material. As part of the procedure the sampling was repeated digitally to eliminate the shreds on the tissue's periphery. ${ }^{10}$

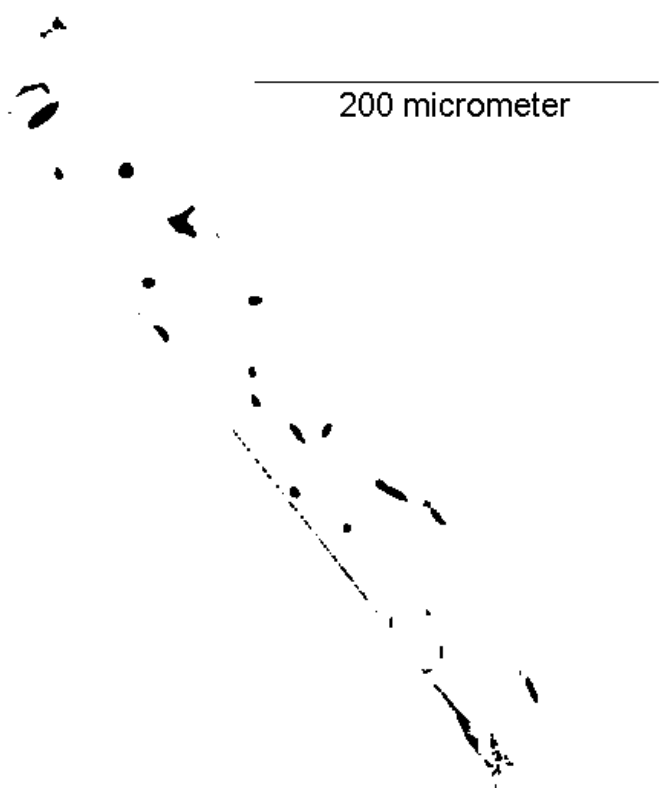

Figure 2. Segmented vessel regions of Figure 1

After the digital punch approach statistical data was gathered about the detected regions. By this process the centroids' coordinates of the detected vessel cross-sections were collected with the matching area parameter, semi-major and semi-minor axis length, the orientation of the major axis and the pixels composing the region's boundary. The statistics of each region were stored uniquely labeled.

After obtaining this information about the regions, the transformed digital images are not required any more in the object reconstruction process, since each region could be represented by the coordinates of its centroid and the collected data. Using this method there is no more need for the original image series during the analysis, the data is stored in a tabular format, and it is much easier to work with the derived results.

\section{Reconstruction of the vessels}

The reconstruction of the blood vessel network was designed as a pipe-work composed by connected, ungula-shaped elements. The ungulas consist of two parallel, circle-shaped planes, and a surface which connects them. The radiuses of the two planes are usually different, and they are derived from the statistics of the detected regions with the centroids' coordinates. To reconstruct the vessel network first the connection has to be established between the discrete elements. This was carried out with an additional identification number. Each region was projected to the image below, and the regions near by the projected centroid were analyzed. If the projected centroid was inside another region the two detected element were joint and both got the same identification number (linking step). If there was no connecting element on the image below, the region got a new identification number. Each region was identified using this method. In case of a bifurcation, the cross-section of the blood vessels has amorphous shape and the statistics about major/minor axis lengths do not describe the geometry of the vessels. These cases must be handled separately, therefore bifurcation detection algorithm was developed. Double 
identification were initiated (a) to enable separate vessel segment handling and (b) to manage them in a complex representation. This method is shown in Figure 3.
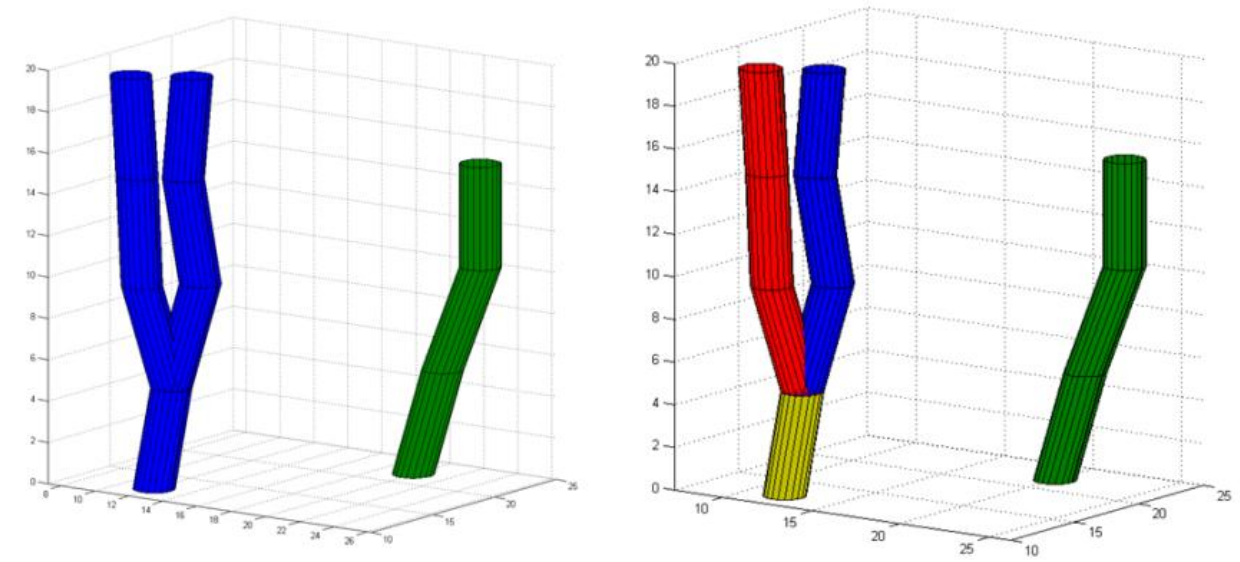

Figure 3. The double element identification: color-code represents unique identifiers for complex structures (left) and separate vessel segments (right)

To reconstruct a blood vessel, the semi-minor axis length and the coordinate of the centroid were used from the statistics of the detected regions. Our method conceives blood vessels as tubular objects. The captured synchrotron images aren't usually perpendicular to vessel axis, so the shape of the section boundaries differs from circle. One can prove, that the semi-minor axis length is the invariant feature which can be taken as the vessels' radius, even if the vessel crosses the image plane almost horizontally. This is shown in Figure 4 where $A$ is the horizontal plane, $B$ is the tilted cross-section, and $R$ means the radius of the vessel.

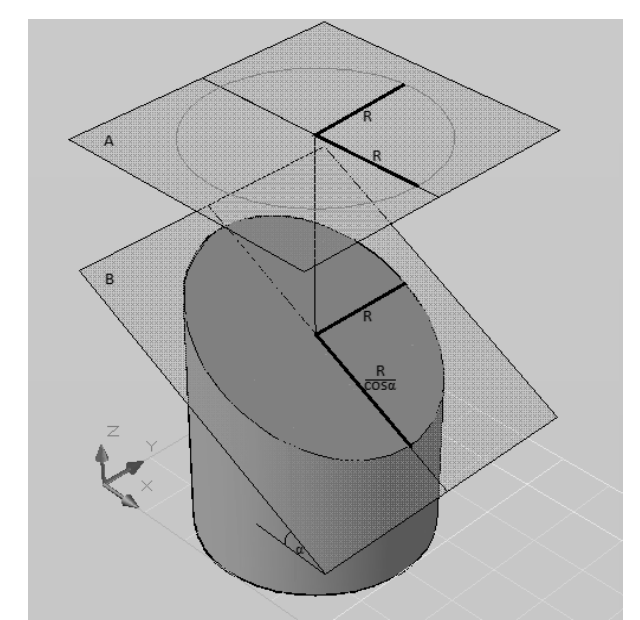

Figure 4. Horizontal and tilted cross-section of a cylinder

\section{Examining the density of vascular structures}

The reconstructed vessel network presented before, enables vascularity analysis. For this the volume of the sample was divided into voxels, and for each voxel the Euclidian distance was calculated to every detected vessel. The minimum of the calculated distances was stored in a three-dimensional array form and to visualize the result, a color tone was attached to each computed distance. Using this method the vascularity of the tissue could be examined in different resolutions, and in every cross-section (in arbitrary directions). Three perpendicular crosssections are shown in Figure 5, where the voxel size is $0.76 \mu \mathrm{m} \times 0.76 \mu \mathrm{m} \times 0.76 \mu \mathrm{m}, 200 \times 200 \times 200$ 
voxels are represented. The gray scale shows the normalized distance from the vessels in the examined part of the tissue and the yellow color shows the vessels.

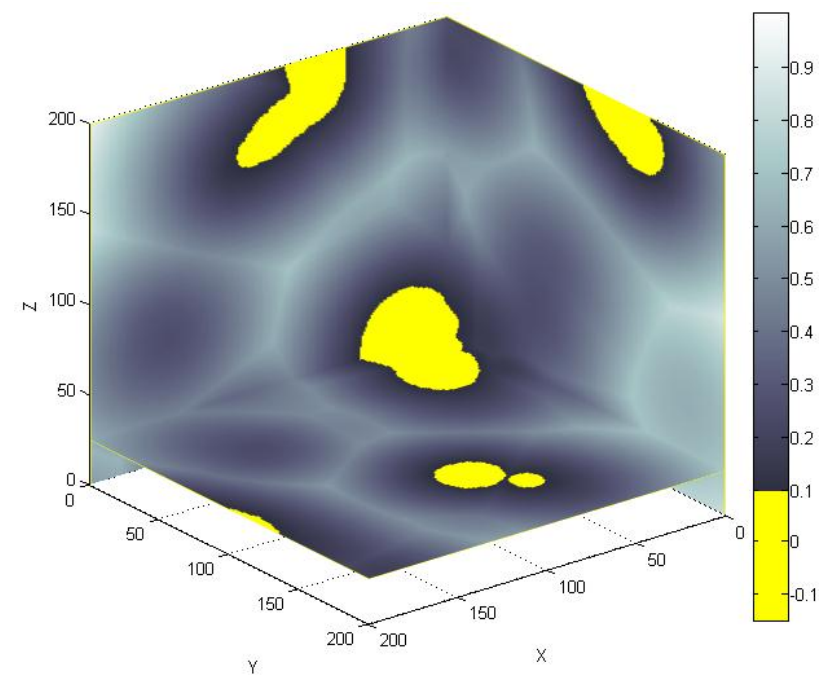

Figure 5. Vascularity in the tissue

\section{Results \& Discussion}

The raw data were transformed into two dimensional images, which could be handled easily by a standard personal computer. The images were segmented and statistics were gathered from the cross-sections of the vessels. The topology of the vessel network was built based on the derived statistical data. The collected data were stored in a structured format to handle each vessel separately. The quality measure of the examined sample is shown in Table 1.

\begin{tabular}{|l|r|}
\hline Number of detected vessel cross-sections: & 12209 \\
\hline Number of detected separate vessels: & 2390 \\
\hline Number of elements in the longest vessel: & 271 \\
\hline Mean number of vessel elements: & 5.11 \\
\hline Global length of detected vessels: & $4639.42 \mu \mathrm{m}$ \\
\hline Length of the longest vessel: & $102.98 \mu \mathrm{m}$ \\
\hline Mean length of the vessels: & $1.94 \mu \mathrm{m}$ \\
\hline Diameter of the greatest vessel: & $43.76 \mu \mathrm{m}$ \\
\hline Mean diameter of the vessels: & $802 \mathrm{~m}$ \\
\hline Volume of the examined sample: & $58818.57 \mu^{3}$ \\
\hline Total vessel volume: & $1742.18 \mu \mathrm{m}^{3} / \mu \mathrm{m}$ \\
\hline Tissue supply (blood supply of the sample per vessel unit) & $\mu \mathrm{m}^{3}$ \\
\hline
\end{tabular}

Table 1. Basic statistics of the analyzed sample

Using the acquired statistics data, the blood vessels could be visualized. Vessels composed from more than nine elements, are plotted using the ungula-based model in Figure 6. 


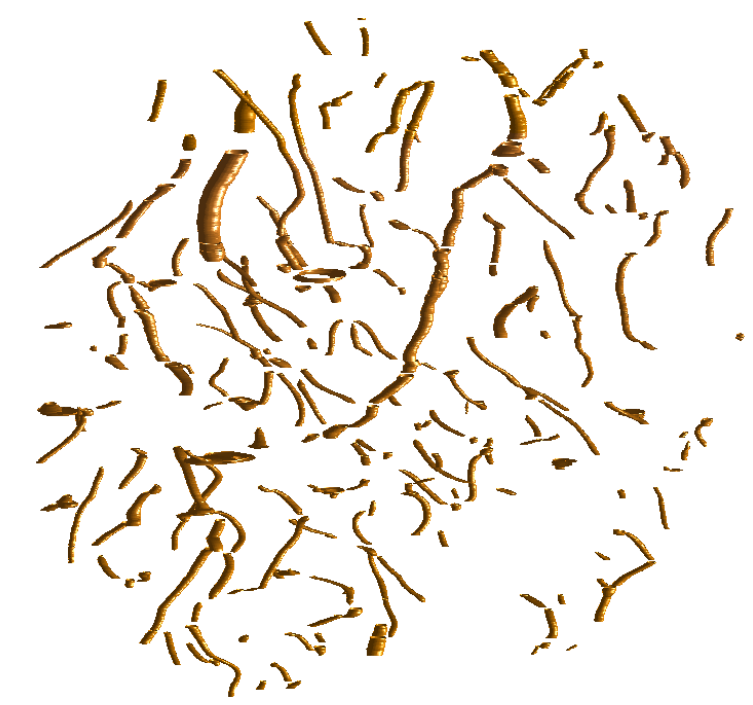

Figure 6. Model of the vessel network using ungula elements

In Figure 7 spheres were plotted instead of ungulas to the centroids with the adherent semi-minor axis length as the radius of the sphere. Significantly larger spheres indicate vessel bifurcations.

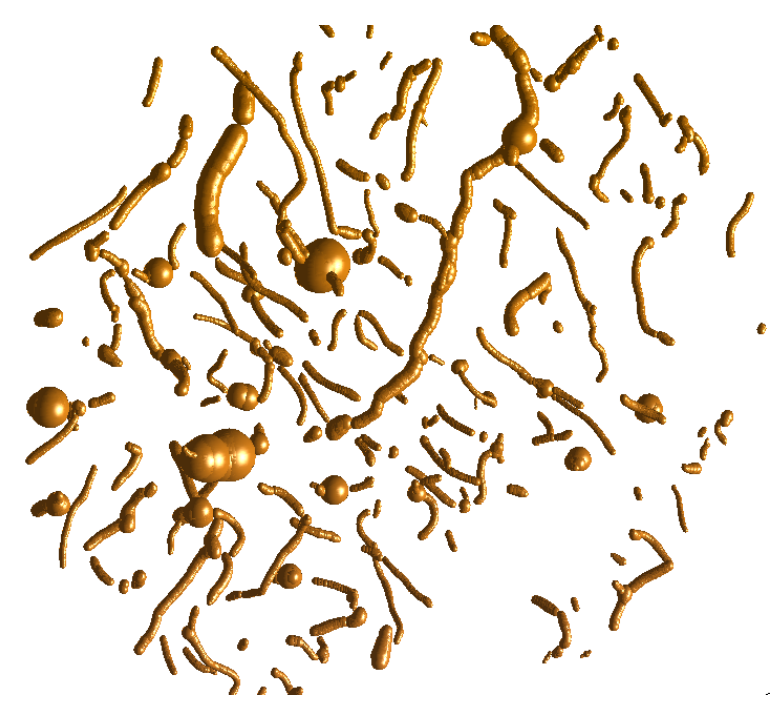

Figure 7. Model of vessel network using sphere elements

\section{Conclusion}

Our method produces the object-model of the micro blood vessel network inside cerebral cortical samples using synchrotron-based tomography. The algorithm detects the vessel sections on two-dimensional tomography images and finds the connection between the separate elements. The algorithm could be used to visualize the micro blood vessels inside the sample, to derive quality measures or to perform quantitative analysis on the vessels. The method needs minimal pre-processing on the biological tissues, and it could provide enough information for the object reconstruction including vessel surface network. An important modification of the present study as compared to previous studies ${ }^{2}$ is that the vessels were not directly stained with contrast agents. The efficacy of perfusing the vascular system with barium-sulfate (standard contrast agent in $\mathrm{x}$ ray examination) or other contrast solutions ${ }^{2}$ is unknown. Here we enhanced the contrast by a non-specific staining of the tissue (proteins and nucleic acids), which leaves the vessel lumen 
completely transparent. Consequently, our procedure is free of labeling artifacts. The data acquired could be the base of a blood vessel surface reconstruction, and by adding physiological information it could be used for simulations of normal and pathological conditions (e. $g$. arteriosclerosis). After analyzing samples from multiple species the difference could be determined automatically by the statistics of the tissues.

\section{REFERENCES}

1. Logothetis NK, Pfeuffer J. On the nature of the BOLD fMRI contrast mechanism. Magnetic Resonance Imaging 2004 Dec;22(10):1517-31.

2. Guibert R, Fonta C, Plouraboué F. Cerebral blood flow modeling in primate cortex. Journal of Cerebral Blood Flow \& Metabolism 2010 Nov;30(11):1860-73.

3. Denk W, Horstmann H. Serial block-face scanning electron microscopy to reconstruct threedimensional tissue nanostructure. PLoS biology 2004 Nov;2(11):e329.

4. Knott G, Marchman H, Wall D, Lich B. Serial section scanning electron microscopy of adult brain tissue using focused ion beam milling. The Journal of Neuroscience 2008 Mar 19;28(12):2959-64.

5. Mizutani R, Takeuchi A, Akamatsu G, Uesugi K, Suzuki Y. Element-specific microtomographic imaging of Drosophila brain stained with high-Z probes. Journal of synchrotron radiation 2008 Jul;15(Pt 4):374-7.

6. Plouraboué F, Cloetens P, Fonta C, Steyer A, Lauwers F, et al. X-ray high-resolution vascular network imaging. Journal of microscopy 2004 Aug;215(Pt 2):139-48.

7. Kapitány K. Object-reconstruction from high-resolution X-ray images (In Hungarian) 2012.

8. Stampanoni M, Groso A, Isenegger A, Mikuljan G, Henein S, Betemps R. Trends in synchrotronbased tomographic imaging: the SLS experience. SPIE Proceedings "Developments in X-ray Tomography V"; 2006; San Diego, CA.

9. ImageJ website. [cited 2013 4. 5.]; http://rsb.info.nih.gov/ij/].

10. Kapitány K. Geometric reduction of high amount histological image data. Conference of Junior Researchers in Civil Engineering; 2012; Budapest.

This study was supported in the framework of the project „Talent care and cultivation in the scientific workshops of BME" project by the grant TÁMOP - 4.2.2.B-10/1-20100009 as well by the SLS (proposal ID 20090952 and 20100156). 